\title{
Evaluation of Ventricular Repolarization Parameters in Patients Admitted to Emergency Department with Electrical Injury
}

\author{
Çaşıt Olgun Çelik ${ }^{1, \star}$, Orçun Çiftci ${ }^{1}$, Murat Muratoğlü ${ }^{2}$ İbrahim Haldun Müderrsioğlu ${ }^{1}$
}

${ }^{1}$ Başkent University Medical School Ankara Training and Research Hospital, Department of Cardiology, Ankara, Turkey

${ }^{2}$ Başkent University Medical School Ankara Training and Research Hospital, Department of Emergency Medicine, Ankara, Turkey

\section{*Correspondence}

drolgunclk09@gmail.com

(Çaşıt Olgun Çelik)

\begin{abstract}
Objective: Prolonged T-peak to T-end (Tp-e), a ventricular repolarization parameter, has been related with ventricular arrhythmias (VAs). Novel electrocardiogram (ECG) parameters of ventricular repolarization have received considerable attention recently. In this study, we sought to investigate ventricular repolarization indexes such as the Tp-e and corrected Tp-e (Tp-ec) intervals, Tp-e/QT, Tp-e/QTc, and Tp-ec/QT ratios in patients with electrical injuries (EIs). Methods: Thirty-six patients diagnosed with EIs and 35 age- and sex-matched healthy control patients were included. Admission ECGs of the EI patients were compared with those of the healthy controls. QT and QTc intervals were measured, and the Tp-e and Tp-ec intervals, Tp-e/QT, Tp-ec/QT, and Tp-e/QTc ratios were then calculated from a 12-lead surface ECG. Results: The QT, Tp-e, Tp-e/QT, Tpe/QTc, Tp-ec/QT were not significantly different between the control group and the EI group $(\mathrm{p}>0.05)$. However, the mean QTc interval was significantly higher in the EI group compared to the control group $(412.81 \pm 25.46$ vs $396.31 \pm 26.47 \mathrm{~ms}$; p:0.009). Furthermore, the Tp-ec and Tp-ec/QT of the EI subgroup with elevated troponin levels significantly differed from those of the EI patients with normal troponin levels ( $\mathrm{p}: 0.033$ and p:0.016, respectively). Conclusions: This retrospective study indicated that patients with EIs tend to have a prolonged QTc interval. Additionally, Tp-ec and Tp-ec/QT, which reportedly designate the tendency for VAs, were significantly higher in the EI patients with elevated troponin I levels than the EI patients with normal troponin levels, suggesting that patients with myocardial injury may be prone to VAs.
\end{abstract}

\section{Keywords}

Electrocardiogram, Electrical Injuries, Repolarization Parameters, QTc

\section{Introduction}

Electric currents may cause many kinds of wounds, especially burn-related injuries of the skin and muscle that likely occur in conjunction with other types of injuries of the heart and vital organs. Electrical injuries (EIs) are a notable problem in developing countries, often caused by electrical devices at work or at home. EIs have been separated into low-voltage $(<1000 \mathrm{~V})$ or high-voltage $(>1000 \mathrm{~V})$ injuries, depending on the power and type of the current. The injuries caused vary according to the voltage of the electrical current, the conductivity of the body, the direction of the current through the body, and the duration of exposure to the current [1]. While burns on the skin may occur in contact areas, some serious injuries of the other vital organs are not discernible. Clinically complicated manifestations of cardiac, neurogenic, and gastrointestinal systems may also be seen as a consequence of EIs [2,3]. Cardiac arrhythmia is a common cause of death from electric shocks, caused by injury to the myocardium, sinoatrial and atrioventricular nodes, electrical conduction pathways, and coronary arteries.
Electrical currents may lead to death, particularly by affecting myocardial depolarization and repolarization $[3,4]$.

Myocardial electrical activity basically consists of two phases: depolarization and repolarization. Ventricular repolarization, which represents a complex period of myocardial electrical activity, is figured on the surface electrocardiogram (ECG) as the interval between the start of the QRS complex and the end of the $\mathrm{T}$ or $\mathrm{U}$ wave (QT). The QT interval and its heart rate-based corrected form, the QTc interval, have been used for monitoring repolarization [5]. However, QT and QTc intervals have been considered to be crude markers of ventricular repolarization. Thus, novel repolarization markers have been described - namely, T-wave peak to end (Tp-e), Tp-e/QT, and Tp-e/QTc. Some studies have recently noted that ventricular repolarization indexes that include the Tp-e interval and the Tp-e/QT ratio are useful markers for detecting malignant cardiac arrhythmias in patients with several clinical manifestations, excluding structural heart disease [6-8]. The Tp-e interval has a powerful 


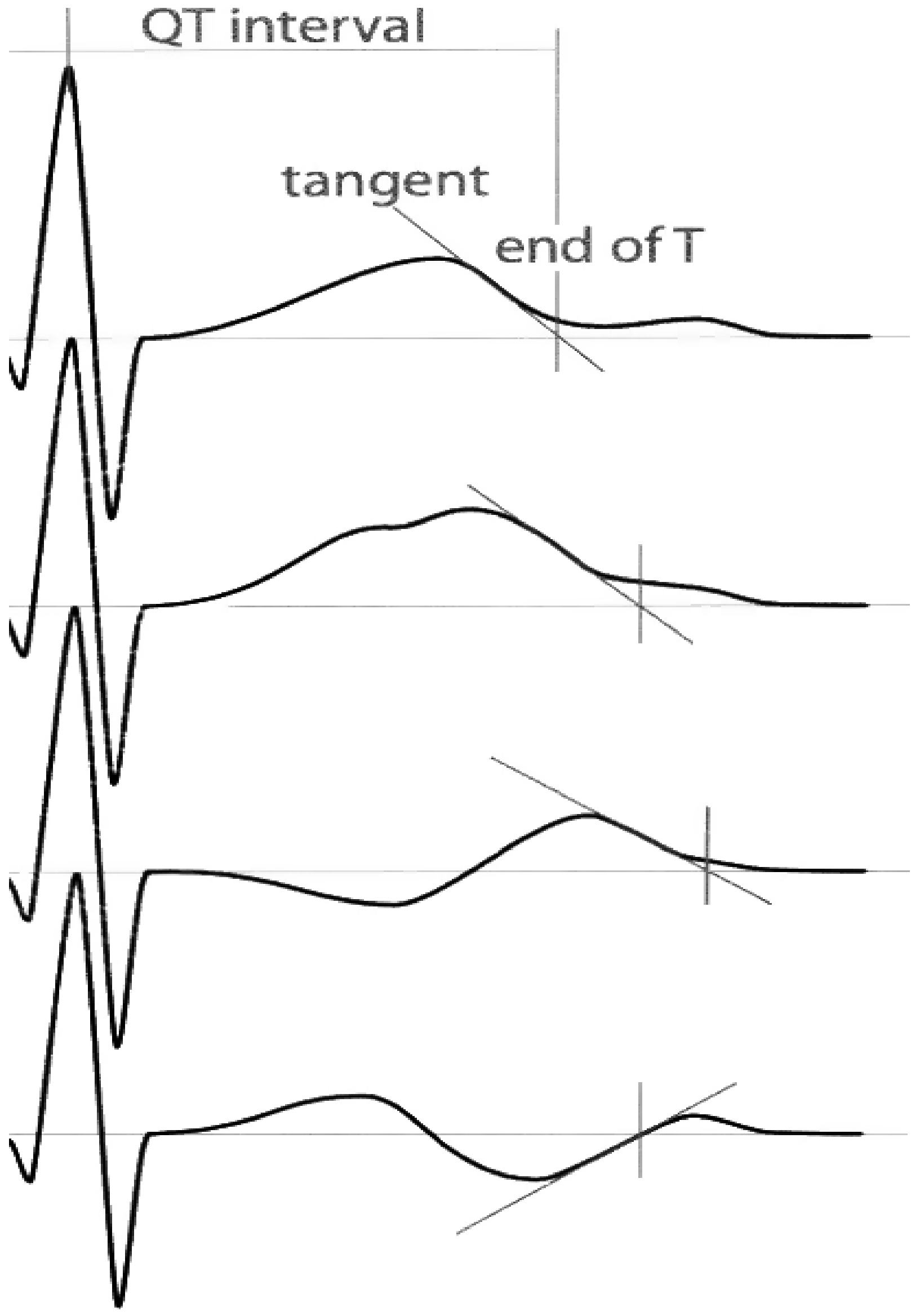

F I G U R E 1. Schematic illustration of the tangent method to define the end of different T-wave forms. Adapted figure reproduced from Postema et al. with permission [16]. 
predictive value for prolonged ventricular repolarization and specifically indicates an increased dispersion of ventricular repolarization. As such, an increased Tp-e interval duration increases the risk of malignant ventricular arrhythmias (VAs); furthermore, the Tp-e interval shows a higher correlation with the distribution of ventricular repolarization than the QT and QTc intervals [9, 10]. Although EIs are known to cause myocardial injury and cardiac arrhythmias, there are scarce data about the outcomes of EIs on novel electrocardiographic repolarization parameters.

The objective of this study was to (1) analyze the ventricular repolarization parameters, including the Tp-e interval, $\mathrm{Tp}-\mathrm{e} / \mathrm{QT}, \mathrm{Tp}-\mathrm{e} / \mathrm{QTc}$ ratios, and heart rate-dependent corrected forms, (2) to compare the Tp-ec and Tp-ec/QT ratios in patients with EIs to healthy subjects, and (3) to create a subgroup analyses of patients with EIs according to their troponin levels.

\section{Methods}

We enrolled 60 consecutive EI patients who presented within the first 2 hours of an EI and who were hospitalized between January 2014 and December 2019 at the Başkent University Ankara Training and Research Hospital. We excluded patients who were under 18 years of age or referred to the emergency room beyond the first 2 hours of receiving an EI; those with a prior history of coronary artery disease, congenital or acquired heart disease, moderate-severe valvular heart disease, severe renal failure, or chronic obstructive pulmonary disease; and those with a prior history of using an antiarrhythmic drug. Patients with complete bundle branch block patterns, atrial fibrillations, pacemaker rhythms, congenitally arrhythmic syndromes, atrioventricular arrhythmias, or traces of electrocardiographic noises that precluded a calculation of the studied repolarization parameters on ECGs were also excluded. We eventually enrolled in the study 36 EI patients with a median age of 28 years, and 35 age- and sex-matched healthy subjects were included as the control group. We recorded demographic and clinical characteristics, the voltage level of the electrical current causing the injury, the laboratory parameters (blood urea nitrogen, creatinine, serum electrolytes, total blood count) on admission, and the third-hour troponin I level. The accepted parameters of a myocardial injury caused by electrical current included those that exceeded the upper limit of our standard laboratory high-specific troponin I level of $0.02 \mathrm{ng} / \mathrm{mL}$. A 12-lead surface ECG was taken from each EI patient at the emergency unit within two hours of admission using a GE Healthcare MAC 2000 (General Electric, Milwaukee) ECG recorder with a paper speed of $25 \mathrm{~mm} / \mathrm{s}$ and voltage of 10 $\mathrm{mm} / \mathrm{mV}$. Using the 12-lead surface ECG, we calculated the QT interval, QTc interval (i.e., the corrected QT interval, modified depending on heart rate by the standard Bazett Formula), Tpe interval, Tp-ec interval, Tp-e/QT ratio, Tp-e/QTc ratio, and $\mathrm{Tp}$-ec/QT ratio. QT measurements were made in all leads, and the longest QT value was accepted, as recommended in the "AHA/ACCF/HRS Recommendations for the Standardization and Interpretation of the Electrocardiogram" [11]. All ECG measurements were made two times by the same cardiologist, blinded to patient identity and condition, and by averaging three consecutive beats. The Intraclass Correlation of Coef- ficient (ICC) method was used for QT and Tp-e intervals to assess the intra-observer variability, and the median ICC of the QT and Tp-e were found to be 0.997 (95\% confidence interval 0.995-0.998, $\mathrm{p}<0.001)$ and 0.989 (95\% CI, 0.982-0.993, $\mathrm{p}<$ $0.001)$, respectively.

Prolonged QTc was defined as $\geq 450 \mathrm{~ms}$ for adult males and $\geq 460 \mathrm{~ms}$ for adult females. In published studies, the Tp-e has been measured using various methods, including manual methods such as the tangent method in V2 and V5, II, the average of measurements in V1-V6, the average of measurements in V4-V6, the maximum Tp-e among V1-V6, and the maximum Tp-e among all leads and the automated method of using the 12-lead surface ECG. We used a typical manual tangent method using precordial V2 and V5 leads as the baseline of the ECG, and T-wave edges were apparently detectable on these two leads to calculate Tp-e; we chose the lead with longest value of Tp-e. It is an even more obscure undertaking to define the end of a $\mathrm{T}$ wave when there is a biphasic, notched, or multiphasic T-wave or a coexisting, faint U-wave form [12], and there are two different ways of calculating the Tp-e interval: namely, the tangent method and the tail method. The existing guidelines recommend the tangent method for T-end assessments, as it is more predictive for mortality rate than the tail method [13-15]. Therefore, the tangent method was used in the present study and was performed manually using a magnifying glass and calipers from the leads V2 and V5, where the Tp-e interval was defined as the time from the peak of the T-wave to its end at a point where it intersects the isoelectric line and the tangent to the downslope of the T-wave (Fig. 1). The Tp-e/QT ratio and the other repolarization parameters were then calculated $[15,16]$. To increase its specificity, the heart rate-dependent correction of the Tp-e (Tp-ec) was calculated using Bazett's formula (Tp$\mathrm{e} / \sqrt{ } \mathrm{RR})[15]$.

\subsection{Statistical analysis}

Statistical analyses were conducted using SPSS 21.0 software (SPSS Inc., IBM, USA). Descriptive statistics included the mean $\pm \mathrm{SD}$ and the median (interquartile range (IQR)) for parametric variables as well as the number and percentage for categorical variables. Quantitative variables were tested for normality of distribution using the Kolmogorov-Smirnov test. The Mann-Whitney U-test was used to compare the quantitative variables, depending on their normality of distribution. The Chi-square test was used to assess categorical variables. To investigate the association between non-normally distributed variables, the Spearman test was used. A p value $<$ 0.05 was accepted as statistically significant.

\section{Results}

From January 2014 to December 2019, 36 of the 60 EI patients who were referred to the emergency department were found eligible for enrollment in this study. Twelve EI patients (33.3\%) were female, and 24 EI patients $(66.7 \%)$ were male. Of the 12 control subjects, $(34.2 \%)$ were male, and $23(65.8 \%)$ were female. The median age of the EI patients was 28 (IQR 18 64) years, while the median age of the healthy controls was 29 
TA B L E 1. Comparison of general demographic, clinical, biochemical data between subjects with and without electrical injury.

\begin{tabular}{|c|c|c|c|}
\hline Variable & EI patients $(n=36)$ & Healthy Control Group $(n=35)$ & p value \\
\hline Age (Years) & $28(18-64)$ & $29(20-61)$ & 0.342 \\
\hline Sex (male) & $24(66.6 \%)$ & $23(65.7 \%)$ & 0.434 \\
\hline Hypertension & $1(2.8 \%)$ & $1(2.9 \%)$ & 0.511 \\
\hline Smoking & $11(30.6 \%)$ & $10(28.6 \%)$ & 0.531 \\
\hline Diabetes Mellitus & $1(2.8 \%)$ & 0 & 0.507 \\
\hline $\mathrm{Na}(\mathrm{mmol} / \mathrm{L})$ & $138.3 \pm 2.4$ & $138.5 \pm 1.4$ & 0.616 \\
\hline $\mathrm{K}(\mathrm{mmol} / \mathrm{L})$ & $4.0(3.6-5.3)$ & $4.1(3.6-4.6)$ & 0.125 \\
\hline $\mathrm{Mg}(\mathrm{mg} / \mathrm{dl})$ & $2.03 \pm 0.26$ & $2.04 \pm 0.18$ & 0.930 \\
\hline $\mathrm{Ca}(\mathrm{mg} / \mathrm{dL})$ & $9.08 \pm 0.45$ & $9.1 \pm 0.21$ & 0.690 \\
\hline Hemoglobin $(\mathrm{g} / \mathrm{dL})$ & $14.75 \pm 2.22$ & $14.9 \pm 1.3$ & 0.796 \\
\hline $\operatorname{WBC}\left(10^{3} / \mu \mathrm{l}\right)$ & $9.3(5.5-33.5)$ & $7.4(4.6-11.0)$ & $<0.05$ \\
\hline Heart Rate (/min) & $82.47 \pm 15.27$ & $75.46 \pm 11.39$ & $<0.05(0.032)$ \\
\hline QT (ms) & $356.42 \pm 30.72$ & $352.23 \pm 24.44$ & 0.527 \\
\hline QTc (ms) & $412.81 \pm 25.46$ & $396.31 \pm 26.47$ & $<0.05(0.009)$ \\
\hline Tp-e (ms) & $77.99 \pm 12.69$ & $76.01 \pm 10.83$ & 0.481 \\
\hline Tp-ec (ms) & $91.05 \pm 16.80$ & $84.91 \pm 11.85$ & 0.079 \\
\hline Tp-e/QT & $0.220 \pm 0.041$ & $0.215 \pm 0.031$ & 0.623 \\
\hline Tp-ec/QT & $0.258 \pm 0.062$ & $0.240 \pm 0.036$ & 0.138 \\
\hline Tp-e/QTc & $0.190 \pm 0.034$ & $0.191 \pm 0.031$ & 0.816 \\
\hline
\end{tabular}

(IQR 20 - 61) years. The basal laboratory findings and clinical characteristics of the EI patients and the healthy controls are given in Table 1, and the comparison of general demographic, clinical, and biochemical data between EI patients with and without troponin I elevation are given in Table 2. There was no significant difference between EI patients and the healthy controls in terms of demographic and clinical characteristics $(p>0.05)$. Twenty-eight EI patients were injured by lowvoltage electricity, and no surgical operation or additional treatment was needed for 26 of the patients, but additional medical treatment was required for the remaining 2 patients of this group. A total of 8 EI patients had operations involving skin grafting as a result of high-voltage electrical burn injuries. The range of troponin I levels of all EI patients was between 0 $\mathrm{ng} / \mathrm{mL}$ and $9.14 \mathrm{ng} / \mathrm{mL}$; this range was between $0.032 \mathrm{ng} / \mathrm{mL}$ and $9.14 \mathrm{ng} / \mathrm{mL}$ for the EI subgroup of high-voltage burn patients. Moreover, a quite significant and positive correlation was observed between the troponin level and the voltage height in the EI group (r:0.736; $\mathrm{p}<0.001)$.

The QT, Tp-e, Tp-e/QT, Tp-e/QTc, and Tp-ec/QT values were not significantly different between the healthy control group and the EI group ( $p>0.05)$ (Table 1); of the EI patients, only 1 female patient was detected with prolonged QTc (464 $\mathrm{msn}$ ), according to our definition. However, the mean QTc interval and heart rate was significantly higher in the EI group compared to the healthy group (412.81 \pm 25.46 vs $396.31 \pm$ 26.47; p:0.009 and $82.47 \pm 15.27$ vs $75.46 \pm 11.39$; p:0.032, respectively). The white blood cell count was significantly higher in the EI group compared to the healthy control group. There was no significant difference between the EI subgroup with normal troponin I levels and the EI subgroup with elevated troponin I levels with respect to heart rate and the studied repolarization indexes, namely QT, QTc, Tp-e, Tp-e/QT, and Tp-e/QTc (p:0.237, p:0.322, p:0.119, p:0.083, p:0.331, and $\mathrm{p}: 0.133$, respectively). However, the Tp-ec and the Tp-ec/QT ratio were significantly higher in the EI subgroup with elevated troponin levels than in the EI subgroup with normal troponin levels (p:0.033 and p:0.016, respectively) (Table 2). Further, the EI subgroup with high troponin levels had a significantly higher white blood cell count than the EI subgroup with normal troponin levels.

\section{Discussion}

This study demonstrated that the QTc interval was significantly prolonged in EI patients compared to the healthy control subjects. Additionally, the Tp-ec and Tp-ec/QT ratio were notably increased in the EI subgroup with elevated troponin I levels compared to the EI subgroup with normal troponin I levels.

EIs are still a major problem worldwide [17]. Various types of VAs can be observed as a result of an EI, ranging from premature ventricular contractions to malignant ventricular tachycardia or ventricular fibrillation. Several hypotheses have sought to explain the underlying mechanism of VAs in EIs, including from damage to the myocardial cell membrane and ion channels, electrolyte imbalance resulting from repolarization abnormalities in cases with increased intracellular potassium and calcium levels, and the direct detriment on the cardiac conduction system by an electrical current [18]. Previous substantive studies have reported seeing nonspecific ST/T wave 
TA B L E 2. Comparison of general demographic, clinical, biochemical data between electrical injury patients with and without troponin I elevation.

\begin{tabular}{lccc} 
Variable & Elevated Troponin I $(\mathbf{n}=\mathbf{1 0})$ & Normal Troponin I $(\mathbf{n}=\mathbf{2 6})$ & p value \\
\hline Age $($ Years $)$ & 36.5 & 25 & 0.041 \\
\hline Sex (male) & $9(90 \%)$ & $15(57.6 \%)$ & 0.069 \\
\hline Hypertension & $1(10 \%)$ & 0 & 0.484 \\
\hline Smoking & $4(40 \%)$ & $7(26.9 \%)$ & 0.353 \\
\hline Diabetes Mellitus & $1(10 \%)$ & 0 & 0.722 \\
\hline Na (mmol/L) & 138 & 138 & 0.794 \\
\hline K (mmol/L) & 4 & 3.95 & 0.958 \\
\hline Mg (mg/dL) & 1.93 & 2.07 & 0.109 \\
\hline Ca $(\mathrm{mg} / \mathrm{dL})$ & 8.8 & 9.2 & 0.087 \\
\hline Hemoglobin $(\mathrm{g} / \mathrm{dL})$ & 15.1 & 14.6 & 0.664 \\
\hline WBC $\left(10^{3} / \mu \mathrm{l}\right)$ & 12.565 & 7.345 & 0.001 \\
\hline Heart Rate $(/ \mathrm{min})$ & 95.5 & 77 & 0.134 \\
\hline QT (ms) & 355.5 & 359 & 0.241 \\
\hline QTc $(\mathrm{ms})$ & 418.5 & 415.5 & 0.337 \\
\hline Tp-e $(\mathrm{ms})$ & 83.53 & 78.45 & 0.126 \\
\hline Tp-ec $(\mathrm{ms})$ & 97 & 87.25 & 0.031 \\
\hline Tp-e/QT & 0.231 & 0.215 & 0.087 \\
\hline Tp-ec/QT & 0.281 & 0.244 & 0.015 \\
\hline Tp-e/QTc & 0.196 & 0.193 & 0.337 \\
\hline
\end{tabular}

changes, bundle branch blocks, first degree atrioventricular block, and sinus bradycardia or tachycardia in EIs [19].

EIs can cause VAs due, particularly, to changes in ventricular repolarization. The Tp-e interval, Tp-e/QT, and Tp-e/QTc ratios are novel repolarization indexes that can be calculated from a 12-lead surface ECG and reportedly reflect the total distribution of repolarization $[16,20]$. Any increase in these parameters has been linked to malignant VAs [21-25].

Although there are several studies about the Tp-e interval, novel ventricular repolarization indexes including Tp-e, Tp$\mathrm{e} / \mathrm{QT}$, and Tp-e/QTc have been inadequately investigated in EI patients. This is one of the rare studies about ventricular repolarization in EI patients that uses the Tp-ec as a novel ECG marker of repolarization (calculated by correcting the Tpe interval by heart rate using Bazett's formula) and the Tpec/QT ratio.

In our study, the Tp-ec and Tp-ec/QT ratio increased significantly in the EI subgroup with elevated troponin I levels compared to the EI subgroup with normal troponin I levels. EIs may affect the myocardium and electrical pathways of the heart. Troponin elevation caused by myocyte death may occur in EIs, depending on the voltage and, especially, the direction of the electrical current through the human body. Troponin elevation is seen variably in different series, ranging from 0 to $72.9 \%[19,26,27]$. However, no data have so far supported the idea that troponin elevation can predict arrhythmias after an EI $[19,28]$. Moreover, the arrhythmogenic effects of an EI are not necessarily fixed to occur due to myocardial necrosis, and arrhythmic complications may well occur before myocardial injury due to the alterations of the electrical impulse and ion channels of the heart affected by the electrical current. This hypothesis is supported by the histopathological observation of the subjects with EIs, proving that the EI causes myofibril degeneration leading to heterogeneity of the conduction system of the heart [18]. Of note, our study demonstrated that EI patients with elevated troponins had altered novel repolarization indexes and thus may be prone to lethal VAs. In our study, there were 10 patients with elevated troponin I levels on admission, most probably through direct myocardial injury by high-voltage electrical current. We noted that novel repolarization indexes were more significantly affected among patients with EIs and elevated troponins, suggesting that the direct myocardial involvement of an EI, and the resulting elevation of troponins, predisposes patients to repolarization abnormalities to a greater degree than patients without myocardial injury and troponin elevation. This may suggest that myocardial necrosis with an altered myocardial architecture that results in homogeneous repolarization may potentially cause fatal VAs. This subject should be clarified by future prospective studies.

\subsection{Limitations}

Our study has several limitations. The major limitation of this retrospective study is that it was conducted in a single center with a small sample size. It would be better to design this study as a prospective study and follow up with patients to compare their latest ECGs after an EI with their initial data or to use a Holter during the hospital stay. Also, we used a magnifying glass to manually measure ECG parameters, Tp-e, and Tp-ec; the use of an automated measurement method might have been 
more effective to reduce intra-observer variability. Moreover, it would be better to compare the EI patients in relation to the level of voltage they experience because there was a lack of difference between the EI group and the healthy subjects in some of the ECG parameters, likely due to the predominance of the EI patients with low-voltage experiences.

\section{Conclusion}

The QTc was found to be significantly more prolonged in patients with EIs than in the control subjects, suggesting myocardial repolarization may also be prolonged in EI patients. Novel repolarization indexes (i.e., Tp-ec and Tp-ec/QT) were more significantly prolonged in EI patients with elevated troponin I levels than those without, suggesting that an injured myocardium may predispose patients to altered myocardial repolarization. Further, larger-scale prospective studies with long-term follow up are necessary to determine the prognostic importance of these repolarization parameters for the prediction of VAs in patients with EI.

\section{ACKNOWLEDGMENTS}

We are grateful to Dr. İbrahim Haldun Müderrisoğlu for his administrative support.

\section{CONFLICT OF INTEREST}

The authors declare that there is no conflict of interest regarding the publication of this article.

\section{AUTHOR'S CONTRIBUTIONS}

ÇOÇ designed and performed the research study. ÇOÇ and OÇ wrote the manuscript. MM provided help to collect data. OÇ analyzed the data. IHM gave his administrative support and supervised the study.

\section{STATEMENT OF COMPETING INTERESTS}

All authors declare that they have no conflicts of interest. No financial support was obtained.

\section{ETHICS APPROVAL}

Ethics committee approval was given as Project No: KA20/43 by the Local Institutional Review Board of the Başkent University Ankara Education and Training Hospital.

\section{REFERENCES}

[1] Smith MA, Muehlberger T, Dellon AL. Peripheral nerve compression associated with low-voltage electrical injury without associated significant cutaneous burn. Plast Reconstr Surg. 2002;109:137-144.

[2] Clark AT, Wolf S. Electrical Injury. JAMA. 2017;318:1198.

[3] Rai J, Jeschke MG, Barrow RE, et al. Electrical injuries: a 30-year review. J Trauma. 1999;46:933-936.

[4] Karadaş S, Gönüllü H, Öncü MR, et al. The effects on complications and myopathy of different voltages in electrical injuries. Turk J Trauma Emerg Surg. 2011;17:349-353.
[5] Cowan JC, Hilton CJ, Griffiths CJ, et al. Sequence of epicardial repolarization and configuration of the T wave. Br Heart J. 1988;60:424433.

[6] Opthof T, Coronel R, Wilms-Schopman FJ, et al. Dispersion of repolarization in canine ventricle and the electrocardiographic $\mathrm{T}$ wave: Tp-e interval does not reflect transmural dispersion. Heart Rhythm. 2007;4:341-348.

[7] Tanriverdi Z, Besli F, Gungoren F. The evaluation of Tp-e interval after transcatheter aortic valve implantation. J Electrocardiol. 2018;51:573.

[8] Yilmaz Coskun F, Elboga G, Altunbas G, et al. Evaluation of ventricular repolarization features with Tp-e, Tp-e/QTc, JTc and JTd during electroconvulsive therapy. J Electrocardiol. 2018;51:440-442.

[9] Opthof T, Coronel R, Janse MJ. Is there a significant transmural gradient in repolarization time in the intact heart?: repolarization gradients in the intact heart. Circ Arrhythm Electrophysiol. 2009;2:89-96.

[10] Panikkath R, Reinier K, Uy-Evanado A, et al. Prolonged Tpeak-to-tend interval on the resting ECG is associated with increased risk of sudden cardiac death. Circ Arrhythm Electrophysiol. 2011;4:441-447.

[11] Rautaharju PM, Surawicz B, Gettes LS, et al. AHA/ACCF/HRS recommendations for the standardization and interpretation of the electrocardiogram: part IV: the ST segment, T and U waves, and the QT interval: a scientific statement from the American Heart Association Electrocardiography and Arrhythmias Committee, Council on Clinical Cardiology; the American College of Cardiology Foundation; and the Heart Rhythm Society. Endorsed by the International Society for Computerized Electrocardiology. JACC. 2009:53;982-991.

[12] Antzelevitch C, Sicouri S, Di Diego JM, et al. Does Tpeak-Tend provide an index of transmural dispersion of repolarization? Heart Rhythm. 2007;4:1114-1116.

[13] Tatlisu MA, Ozcan KS, Gungor B, et al. Can the T-peak to T-end interval be a predictor of mortality in patients with ST-elevation myocardial infarction? Coron Artery Dis. 2014;25:399-404.

[14] Porthan K, Viitasalo M, Toivonen L, et al. Predictive value of electrocardiographic T-wave morphology parameters and T-wave peak to T-wave end interval for sudden cardiac death in the general population. Circ Arrhythm Electrophysiol. 2013;6:690-696.

[15] Rosenthal TM, Masvidal D, Abi Samra FM, et al. Optimal method of measuring the T-peak to T-end interval for risk stratification in primary prevention. Europace. 2018;20:698-705.

[16] Postema PG and Wilde AA. The Measurement of the QT Interval. Current Cardiology Reviews. 2014;10:287-294.

[17] Nursal TZ, Yildirim S, Tarim A, et al. Burns in southern Turkey: electrical burns remain a major problem. J Burn Care Rehabil. 2003;24:309-314.

[18] Fineschi V, Karch SB, D'Errico S, et al. Cardiacpathology in death from electrocution. Int J Legal Med. 2006;120:79-82.

[19] Searle J, Slagman A, Maaß W, et al. Cardiac monitoring in patients with electrical injuries. An analysis of 268 patients at the Charité Hospital. Dtsch Arztebl Int. 2013;110:847-853.

[20] Kors JA, Ritsema van Eck HJ, van Herpen G. The meaning of the Tp-Te interval and its diagnostic value. J Electrocardiol. 2008;41:575-580.

[21] Gupta P, Patel C, Patel H, et al. T(p-e)/QT ratio as an index of arrhythmogenesis. J Electrocardiol. 2008;41:567-574.

[22] Castro Hevia J, Antzelevitch C, Tornes Barzaga F, et al. Tpeak-tend and Tpeak-to end dispersion as risk factors for ventricular tachycar$\mathrm{dia} /$ ventricular fibrillation in patients with the Brugada syndrome. J Am Coll Cardiol. 2006;47:1828-1834.

[23] Zhao X, Xie Z, Chu Y, et al. Association between Tp-e/QT ratio and prognosis in patients undergoing primary percutaneous coronary intervention for ST-segment elevation myocardial infarction. Clin Cardiol. 2012;35:559-564.

[24] Hetland M, Haugaa KH, Sarvari SI, et al. A novel ECG- index for prediction of ventricular arrhythmias in patients after myocardial infarction. Ann Noninvasive Electrocardiol. 2014;19:330-337.

[25] Karaman K, Altunkaş F, Cetin M, et al. New markers for ventricular repolarization in coronary slow flow: Tp-e interval, Tp-e/QT ratio, and Tp-e/ QTc ratio. Ann Noninvasive Electrocardiol. 2014;20:338-344.

[26] Karataş MB, Onuk T, Güngör B, et al. Assessment of Electrocardiographic Parameters in Patients With Electrocution Injury. J electrocardiol. 2015;48:809-814. 
[27] Jae Hyuk Choi, Donghoon Han, Si-Hyuck Kang, et al. Retrospective study of prognosis and relating factors of cardiac complications associated with electrical injuries at a single centre in Korea. BMJ. 2019;9:e028741.

[28] Pawlik AM, Lampart A, Stephan FP, et al. Outcomes of electrical injuries in the emergency department: a 10-year retrospective study. Eur J Emerg Med. 2016;23:448-454.
How to cite this article: Çaşıt Olgun Çelik, Orçun Çiftci, Murat Muratoğlu, İbrahim Haldun Müderrsioğlu. Evaluation of Ventricular Repolarization Parameters in Patients Admitted to Emergency Department with Electrical Injury. Signa Vitae. 2020;16(2):182-188. doi:10.22514/sv.2020.16.0076. 